\title{
INVESTIGACIONES
}

\section{Leer y escribir en la universidad: una experiencia desde una concepción no instrumental ${ }^{*}$}

\author{
Reading and writing at University: An experience from a \\ non instrumental conception
}

\author{
Andrea Torres Perdigón \\ Departamento de Lenguas, Pontificia Universidad Javeriana \\ Universidad EAN, Bogotá, Colombia \\ Telf.: (57) 3208320. Correo electrónico: atorresp@ javeriana.edu.co
}

\begin{abstract}
RESUMEN
Este artículo trata de las dificultades de lectura y escritura que experimentan los estudiantes universitarios actualmente, y presenta los resultados de una investigación sobre este tema llevada a cabo en la Universidad EAN, de Bogotá, en 2015. En el estudio se diseñaron estrategias de fortalecimiento para dos cursos iniciales de lectura y escritura destinados a estudiantes de todas las carreras, además de pruebas de entrada y de salida. Estas actividades de investigación se fundaron en una concepción no instrumental de estas habilidades y el artículo argumenta cómo esta, al igual que el énfasis en la conciencia de los hábitos, las prácticas, las herramientas de la lectura crítica y los rasgos de la escritura argumentativa académica, son fundamentales para el desempeño crítico y argumentativo de los estudiantes universitarios.
\end{abstract}

Palabras clave: alfabetización académica, lectura crítica, escritura académica, argumentación, pensamiento crítico.

\section{ABSTRACT}

This article deals with the reading and writing difficulties that undergraduate students experience nowadays. It presents the research results of an investigation that was undertaken in 2015 at EAN University, in Bogota. During the study, some strengthening strategies were designed for two basic reading and writing courses for students across all the disciplines, as well as a pre-test and a post-test. These research activities were created from a noninstrumental point of view of reading and writing skills. The article supports this conception, and focuses on the consciousness of habits, practices, critical reading tools, and the characteristics that academic writing constitutes a basis for the development of critical thinking and argumentation skills of undergraduate students.

Key words: literacy, critical reading, academic writing, argumentation, critical thinking.

Este proyecto se desarrolló dentro de la línea de investigación Lengua Española y Ciencias del Lenguaje, de la Facultad de Humanidades y Ciencias Sociales de la Universidad EAN. El proyecto fue financiado por esta Universidad y el equipo estuvo conformado por los siguientes investigadores: Andrea Torres Perdigón, Bibian Paola Fernández Luna y Carolina Niño Pantoja. 
“[...] las habilidades básicas (leer, escribir, contar) no pasan del nivel de la primaria: no son conocimientos propiamente hablando, sino condiciones de su adquisición"”.

François Rastier, Apprendre pour transmettre (Rastier, 2013, p. 31)

\section{INTRODUCCIÓN}

Durante las últimas tres décadas, se han llevado a cabo distintas investigaciones acerca de las dificultades de lectura y escritura de los estudiantes universitarios. La investigación y el acercamiento a esta problemática se han desarrollado en particular en el ámbito anglosajón y, de manera más reciente, en el campo de las universidades hispanohablantes. Según Jones, Torne y Street (1999), en el contexto de la lengua inglesa "Con la expansión de la educación superior en una cantidad de países (el Reino Unido, Estados Unidos de América, Sudáfrica), la atención se ha centrado cada vez más en los 'problemas' que los estudiantes enfrentan al encontrarse con los requerimientos de escritura de la academia" (Jones et al., 1999, p. xv). En efecto, la expansión de la universidad en distintos países, así como el intento de reducir las cifras de deserción en la educación superior han hecho de las dificultades de los estudiantes, especialmente de aquellos que inician su carrera universitaria, un foco de atención para los docentes e investigadores.

En distintas universidades norteamericanas, por ejemplo, la figura institucional de los centros de escritura (writing centers) empezó a desarrollarse de manera sólida en la década de los ochenta, así como las redes de colaboración entre estos, como la Internacional Writing Centers Association (IWCA), fundada en 1983 (IWCA, 2015). Estos centros sirven como apoyo a los estudiantes de múltiples disciplinas en todas las actividades relacionadas con los procesos de escritura dentro de la universidad. En el contexto latinoamericano, distintas universidades han comenzado también a implementar este tipo de figura y a crear redes con el propósito de avanzar en investigación y en estrategias de mejora. El objetivo de este tipo de centros es apoyar múltiples procesos de formación y aprendizaje vinculados con la escritura de los estudiantes tanto de carreras profesionales como de estudios de posgrado. Por ejemplo, en el año 2014 se fundó la Red Latinoamericana de Centros y Programas de Escritura (RLCPE), que actualmente organiza congresos para compartir distintas experiencias de este tipo de centros en Latinoamérica (RLCPE, 2014).

Además de la estrategia específica de estos centros, la enseñanza y la investigación acerca de las prácticas de lectura y escritura de los estudiantes universitarios han sido pensadas a través de conceptos como cultura escrita, literacidad (literacy) o alfabetización académica. En el ámbito hispanohablante, distintas publicaciones de Paula Carlino (Carlino, 2005, 2011, 2013) han tenido una influencia significativa en las aproximaciones investigativas a esta problemática, primordialmente a partir del concepto de alfabetización académica. Según esta autora, esta se entiende como el

[...] proceso de enseñanza que puede (o no) ponerse en marcha para favorecer el acceso de los estudiantes a las diferentes culturas escritas de las disciplinas. Es el intento

Las traducciones de las citas son personales. 
denodado por incluirlos en sus prácticas letradas, las acciones que han de realizar los profesores, con apoyo institucional, para que los universitarios aprendan a exponer, argumentar, resumir, buscar información, jerarquizarla, ponerla en relación, valorar razonamientos, debatir, etcétera, según los modos típicos de hacerlo en cada materia. (Carlino, 2013, p. 370).

Se trata entonces de concebir la alfabetización académica como un proceso que busca facilitar el ingreso de los estudiantes universitarios a cada una de las culturas escritas disciplinares. Esta idea de "facilitar" parte de la constatación reiterada de una serie de dificultades asociadas al contacto con la cultura escrita de cada disciplina. La entrada a la vida académica universitaria no es entonces pensada, desde esta perspectiva, como una etapa o un paso evidente de una fase a otra de formación, sino como un cambio de prácticas que, al ser sustancial, puede necesitar una serie de procesos de apoyo paralelos. Estas dificultades han sido expuestas con especial énfasis en el continente americano, aunque trabajos como el de Björk y otros (2003) mencionan otras experiencias en el contexto de las universidades europeas. En cualquier caso, se trata de un fenómeno ampliamente difundido en distintos países, en diversos sistemas educativos públicos y privados, y en múltiples lenguas y culturas, aspecto que puede responder a causas de múltiples órdenes y de difícil esclarecimiento, pero cuya constatación merece ser explícita.

\section{EL DISEÑO DE LA INVESTIGACIÓN}

La universidad colombiana se encuentra, sin duda, ante el mismo fenómeno y cada institución intenta estudiar y comprender estas dificultades desde distintas iniciativas institucionales. La Universidad EAN ${ }^{2}$ en Bogotá, donde se desarrolló la investigación que aquí presentamos, es una institución privada que ofrece programas de pregrado, maestría y doctorado, principalmente en áreas de administración, finanzas y ciencias económicas; de humanidades y ciencias sociales y de ingenierías. Todos los programas de la Universidad EAN tienen énfasis en el emprendimiento, en consonancia con una filosofía institucional que busca vincular la formación integral y el fomento de la actitud emprendedora, como dos ejes principales de las mallas curriculares.

En el contexto de esta universidad, todas las carreras profesionales cuentan con dos asignaturas dentro de la malla curricular que corresponden al desarrollo de habilidades de lectura, escucha, escritura y oralidad. Estas asignaturas se llaman Competencias Comunicativas I y II, y son vistas por los estudiantes de pregrado durante los primeros semestres de su formación universitaria - generalmente en los dos semestres del primer año de carrera- Estos cursos cuentan con estudiantes de todas las carreras, en grupos de 25 a 30 individuos, y la intensidad de cada una es de seis horas semanales durante nueve semanas consecutivas de clase; es decir, cada curso consiste en 54 horas presenciales durante nueve semanas, periodo que coincide con lo que en esta universidad se llama un ciclo académico.

A pesar de la existencia de estos cursos, las dificultades de los estudiantes relacionadas con la lectura y la escritura han sido identificadas por la EAN recientemente, en especial en

Sigla que significa Escuela de Administración de Negocios. 
una publicación acerca de la lectura crítica y de la importancia del desarrollo transversal de las competencias comunicativas en múltiples asignaturas, no solo en aquellas que están a cargo de los docentes de lengua española (Benavides \& Sierra, 2013). Esta constatación llevó a la formulación del proyecto de investigación "Lectura crítica y escritura argumentativa en la Universidad EAN: estrategias de fortalecimiento y apoyo", que se desarrollará en dos fases: una en 2015, cuyos resultados presentamos en este artículo, y otra que se llevará a cabo en 2016. El problema de investigación se centró en determinar cómo podría la Universidad EAN mejorar los procesos de lectura y de escritura de sus estudiantes y futuros profesionales. A partir de este problema, surgieron múltiples interrogantes: ¿qué estrategias generales es posible implementar para apoyar el desarrollo de la alfabetización académica? ¿Qué actividades dentro del aula es posible realizar para que el trabajo de lectura y escritura de los estudiantes de pregrado sea más sólido en sus carreras? ¿Cómo es posible delimitar un campo tan amplio — como las prácticas de lectura y escritura en la universidad - para realizar un proyecto de investigación que pueda dar cuenta de cambios concretos y observables?

Con respecto a este último interrogante, y antes de pensar en un proyecto más amplio que involucre el trabajo con todas las disciplinas de la universidad y, por lo tanto, con varios docentes de áreas diversas, se pensó en limitar el foco de atención del estudio a dos aspectos específicos: la lectura crítica y la escritura argumentativa. Esta delimitación temática se justifica porque, por una parte, son los temas reiterativos en las evaluaciones de los docentes, y por otra coinciden con el énfasis de la prueba estatal "Saber Pro", examen estandarizado que busca medir la calidad de la educación superior en Colombia. Así, el centro de atención es la lectura crítica y la escritura argumentativa, más que la alfabetización académica en términos generales.

Luego de esta delimitación, se planteó una hipótesis global: dos iniciativas pueden contribuir a la mejora de las dificultades relacionadas con la lectura crítica y la escritura argumentativa. Una primera iniciativa consiste en fortalecer las herramientas y los cursos ya existentes en la EAN, es decir, las asignaturas de Competencias Comunicativas I y II; la segunda iniciativa, pensada para la segunda fase del proyecto, implica actividades nuevas de apoyo a través de un programa de lectura y escritura que, además de asesorías para los estudiantes, pueda encargarse de la ayuda a toda la comunidad académica en cuestiones relacionadas con este tema. Este artículo da cuenta de esta primera fase, es decir, de las actividades de apoyo a las asignaturas de Competencias Comunicativas I y II, realizadas durante el tercer ciclo académico de 2015.

El propósito de esta primera fase — que es la que aquí presentamos- era mejorar la lectura crítica y la escritura argumentativa dentro del aula en estas dos asignaturas, por medio de actividades de apoyo. Con este fin, se diseñaron secuencias didácticas nuevas para los syllabus (o programas) de estas asignaturas, por un lado, e instrumentos de recolección de los datos, por otro. Las secuencias buscan incorporar actividades de apoyo para la lectura crítica o la escritura argumentativa de manera específica, mientras que los instrumentos de recolección permiten obtener una visión global de todos los grupos de Competencias Comunicativas, al unificar los criterios de evaluación de la lectura y la escritura. A continuación presentaremos el marco teórico en el que el equipo de investigadores se apoyó para la concepción general del estudio, el diseño de las secuencias, la recolección y el análisis de datos y la propuesta de la metodología de investigación que se describirá más adelante. 


\section{MARCO TEÓRICO Y CONCEPTUAL}

El marco teórico de esta investigación contempla varias disciplinas y perspectivas dentro de las ciencias humanas. Si bien el enfoque global es constructivista (Vigotsky, 1973) y comprende también una orientación pragmática — que se refleja en la preocupación por dificultades y alternativas concretas-, las herramientas teóricas provienen de los estudios literarios, del análisis del discurso, de la filosofía, de la lingüística y de las ciencias de la educación. Esta variedad de campos disciplinares se debe a lo siguiente: las habilidades que están en juego al estudiar la lectura crítica y la escritura argumentativa de los estudiantes universitarios no tienen solo componentes relacionados con el manejo de la lengua española, sino que se relacionan también con el pensamiento crítico; con la capacidad de reconocer discursos y géneros; con el manejo retórico, lógico y argumentativo; y con la condición de posibilidad para el aprendizaje de cualquier contenido, como señala el epígrafe del lingüista François Rastier. En otras palabras, cuando se habla de facilitar el proceso para leer críticamente y para argumentar por escrito, se ven involucradas muchas habilidades complejas que no tienen que ver exclusivamente con el manejo de la lengua materna, que en este caso es el español. Por esta razón, se consideró insuficiente limitar el marco teórico a disciplinas como la lingüística aplicada o a las teorías del aprendizaje. La problemática de las dificultades en lectura y escritura de los universitarios implica, desde nuestra perspectiva, un conjunto de habilidades y de conocimientos que es irreductible al simple manejo correcto y gramatical de la lengua materna, cualquiera que esta sea, o a una aproximación al problema únicamente lingüística o discursiva: las relaciones entre la lengua, el pensamiento, el aprendizaje, la crítica, la argumentación y los discursos disciplinares son más complejas de lo que cualquier solución solamente pragmática o unidimensional pueda contemplar.

Esta complejidad demandó entonces una primera aproximación desde múltiples disciplinas. De esta manera, elementos de la teoría de los géneros literarios (Combe, 1992), de la lingüística textual (Adam, 2011a, 2011b), de la nueva retórica (Perelman, 1977, 1997; Perelman \& Olbrechts-Tyteca, 1989) y del constructivismo (Vigotsky, 1973) fueron parte del fundamento teórico de las actividades propuestas. Es importante señalar que, además de las disciplinas tradicionales que hemos mencionado - los estudios literarios, el análisis del discurso, la lingüística, las ciencias de la educación y la filosofía- y que han tratado cuestiones relacionadas con los discursos, las ideologías, los géneros, los tipos de textos, la retórica, la lógica, la argumentación y, en general, una concepción no instrumental de la lectura y la escritura, han surgido recientemente algunas propuestas teóricas sobre la alfabetización académica que configuran una suerte de disciplina naciente. Es el caso de los estudios de Carlino (Carlino, 2005, 2011, 2013; Carlino \& Fernández, 2014) o de Cassany (2006), en el contexto hispánico, o de Jones y otros (1999) y Russell (1990, 2013) e, incluso, de una importante bibliografía sobre lo que en el contexto anglosajón se denomina academic writing. Cuando hacemos alusión a una concepción no instrumental, nos referimos a perspectivas que evitan el énfasis en la función y el uso de estas habilidades como instrumento o medio para otros fines externos y concretos, sino que, por el contrario, se han aproximado a ellas contemplando principalmente su articulación intrínseca y extrínseca, así como sus características. Ahora bien, frente a esta multiplicidad de campos disciplinares y de teorías dentro de cada uno, el estudio se centró en tres ejes conceptuales: la alfabetización académica, la lectura crítica y la argumentación. 
De las teorías recientes acerca de la lectura y la escritura contemporáneas se tomaron entonces algunos conceptos para precisar los objetivos de la investigación, entre ellos el de alfabetización académica, cuya definición hemos citado anteriormente según los trabajos de Paula Carlino. Ahora bien, para esta autora, la integración a la cultura escrita de cada disciplina no depende exclusivamente de cursos específicos relacionados con estas dos habilidades en su sentido más básico: "Los especialistas en lengua, psicología, en análisis del discurso, en educación, incluso en escritura, raramente lograrán solos enseñar a leer o a escribir en biología porque no forman parte de su comunidad letrada. Pueden ayudar a tomar conciencia del funcionamiento del lenguaje en ese ámbito, lo cual es distinto de enseñar a comprender y producir sus textos" (Carlino, 2013, p. 361). De esta manera, Carlino propone pensar la lectura y la escritura en la universidad como una serie de prácticas que están ligadas a cada disciplina particular. Es decir, plantea salir de la concepción básica de estas habilidades para acercarse a una comprensión discursiva y disciplinar, más allá del carácter fundamental que estas competencias tienen dentro del proceso de aprendizaje de cualquier campo. De ahí que, según ella, "Enseñar géneros académicos es, entonces, posibilitar que los alumnos se incluyan en situaciones discursivas típicas de comunidades especializadas, según propósitos, significados y valores compartidos" (Carlino, 2013, p. 362).

Frente a esta propuesta de una alfabetización académica ligada a cada disciplina universitaria, y aunque compartimos esta concepción, se optó por un primer acercamiento desde el carácter básico o transversal de la lectura y la escritura en la universidad, debido al tipo de dificultades más recurrentes de los estudiantes de la Universidad EAN. Como lo señala Russell (2013), hay dos concepciones de la escritura académica: una transversal y otra especializada. Estas han sido identificadas por él como una de las contradicciones de la enseñanza de la escritura en el sistema estadounidense y de cómo el movimiento de escribir en el currículo se situaba frente a una concepción de escritura exclusivamente transversal: "Así, el movimiento de escribir a través del currículo se formó (Russell 2002) como una respuesta a las limitaciones a la noción de la escritura como transversal y de la habilidad de escribir como algo fácilmente transferible" (Russell, 2013, p. 164). Frente a estas dos concepciones, esta investigación, más que establecer una división entre ambas, definió que las estrategias de mejora tendrían que tener en cuenta ambos sentidos de la escritura académica: uno básico y transversal, y otro disciplinar y especializado.

Por esta razón, se decidió en esta primera fase fortalecer y mejorar los cursos básicos de Competencias Comunicativas, y preparar así, desde algunos elementos esenciales de la distancia crítica en la lectura y de la capacidad de argumentar por escrito, el futuro trabajo conjunto con docentes de otras disciplinas; es decir, el trabajo que correspondería a la alfabetización académica, tal como la entiende Carlino actualmente. Dicho de otra manera, era importante fortalecer en un primer momento el nivel básico de estas habilidades, antes de interactuar con los docentes de cada disciplina en la universidad y con los géneros textuales específicos. Lo anterior parte de una convicción explícita en el equipo de investigadores: sin un trabajo previo de revisión y de apoyo de las prácticas de lectura y escritura en la universidad, desde su carácter fundamental y básico, el trabajo posterior junto con docentes de otras áreas podría ser poco fructífero. La indiferencia frente a la importancia de la lectura crítica y de la escritura argumentativa en los estudiantes hace, desde nuestra perspectiva, que la iniciación en los discursos disciplinares sea más difícil. De esta manera, el concepto de la alfabetización académica se trabajó, al menos en esta primera fase, desde el carácter básico de las habilidades de lectura y escritura o, en términos de Russell (2013), desde su sentido transversal. 
El segundo eje conceptual fue el de la lectura crítica. En este punto, las distinciones de Cassany (2006) entre tres formas distintas de lectura (una literal o que lee las líneas, otra de comprensión o que lee entre líneas y otra crítica que lee tras las líneas) fueron tenidas en cuenta para definir el carácter crítico de la lectura. Se trata entonces de un reconocimiento del lugar de enunciación y de la ideología que domina los textos en cualquier ámbito, más allá de su carácter literal y de las relaciones semánticas que implican la comprensión de un texto. Más que el entusiasmo explícito de Cassany frente a las nuevas formas de lectura y escritura relacionadas con las tecnologías de la información y la comunicación (TIC), compartimos el concepto de la lectura crítica como un reconocimiento de los elementos ideológicos de un texto — esté en el soporte en el que esté- y la posibilidad para un lector de establecer una distancia frente a ellos como discursos que coexisten con otros y que, en esa medida, no tienen un valor de verdad absoluta. Este concepto de lectura crítica fue el que se adoptó para el diseño de las distintas actividades.

Por último, con respecto al tercer eje conceptual, la escritura argumentativa, los trabajos de Chaïm Perelman (Perelman, 1997; Perelman \& Olbrechts-Tyteca, 1989) y su rescate de la retórica fueron determinantes para definir el ejercicio argumentativo de los estudiantes. Este se definió como una actividad que, no por alejarse de los procedimientos lógicos formales, deja de ser un tipo de racionalidad que puede y debe estudiarse en su especificidad, así como en su relación con la capacidad de convencer a otros y de defender posturas e ideas determinadas. De esta forma, la escritura argumentativa se fundamentó como un ejercicio relacionado con la nueva retórica de Perelman y con procedimientos rigurosos para el establecimiento de razones.

Estos tres ejes conceptuales fueron el marco para el diseño de las secuencias didácticas que se desarrollaron en algunos grupos de Competencias Comunicativas I y II en la EAN, así como para la concepción de instrumentos para observar y comparar las formas de lectura literal, comprensiva y crítica. A continuación explicaremos en detalle el camino que siguió el estudio y la articulación de las distintas actividades.

\section{METODOLOGÍA: LA EXPERIENCIA EN AULA Y LA EVALUACIÓN}

La primera fase de este estudio se desarrolló a partir de una orientación mixta (Creswell, 2013); es decir, contempló elementos cuantitativos y otros cualitativos. Sin embargo, dado que se trata de una investigación de competencias que tienen que ver con la expresión de la argumentación por escrito y de la habilidad de leer críticamente, el énfasis durante el desarrollo de las actividades en las aulas fue de carácter principalmente cualitativo. Si bien algunos datos numéricos proporcionaron información general acerca de los grupos y del desempeño académico de los estudiantes, el propósito principal era analizar la observación de los cambios a partir de las secuencias didácticas diseñadas e implementadas en las aulas. La observación de estas secuencias y la recolección de datos obtenidos fueron compartidas por los investigadores. Posteriormente se analizaron los datos numéricos de todos los estudiantes que cursaron Competencias Comunicativas I y II durante el tercer ciclo académico de 2015.

Los datos numéricos se derivaron de los resultados obtenidos por los estudiantes en las pruebas de escritura y lectura desarrolladas al inicio y al final de los cursos de Competencias Comunicativas I y II. Estos resultados fueron tabulados y depurados; inicialmente se realizó un análisis estadístico descriptivo, consistente en la identificación de medidas como media, 
desviación estándar, mínimos y máximos (para los resultados totales) y de frecuencias y porcentajes de acierto para las preguntas de la prueba de lectura. Para comprobar la existencia de diferencias significativas entre los grupos, se realizaron análisis multivariados como la prueba T, Anova y Chi-cuadrado, con el apoyo del programa SPSS.

Dentro de las múltiples orientaciones de investigación en las ciencias humanas y de posibles métodos de investigación cualitativa, se optó por el enfoque de la investigaciónacción, según las pautas de McNiff (2013) y de Latorre (2008). Este enfoque se eligió porque permitía reflexionar sobre las prácticas en el aula con el propósito de mejorarlas. A partir de los ciclos de planificación, acción, observación y reflexión (Latorre, 2008), característicos de esta metodología, era posible intervenir en las prácticas en aula y, simultáneamente, recolectar datos de la observación de esas intervenciones. La elección de la investigación-acción hacía posible definir métodos para una recolección de datos precisa, sistematizar las prácticas en el aula y, así, dar cuenta del posible cambio a partir de las secuencias implementadas. De igual manera, el énfasis en las prácticas en la clase más que en las dificultades de los estudiantes de manera abstracta hizo posible que las secuencias didácticas tuvieran un carácter concreto y práctico, aspecto útil para observar los resultados y para poder emplear las secuencias en otros cursos.

Por otro lado, y además de la investigación-acción como metodología, se optó por completar las secuencias didácticas con una estrategia inspirada en el cuasiexperimento (Creswell, 2013). Esta consistió en diseñar una prueba de entrada y una de salida para los dos niveles de Competencias Comunicativas. Estas pruebas fueron presentadas por los estudiantes de todos los grupos (en total diez grupos del nivel I y trece grupos del nivel II). De este total de grupos, las secuencias didácticas se usaron solo en tres, que funcionaron de manera análoga a grupos experimentales: dos del nivel I y uno del nivel II. Se diseñaron entonces dos secuencias didácticas para Competencias Comunicativas I, y una para el nivel II. Cabe aclarar que no se trababa de usar este método para falsear ninguna hipótesis en particular, como lo exigiría en rigor el cuasiexperiemento (Campbell \& Stanley, 2005), ni para probar el resultado de las secuencias didácticas diseñadas con datos cuantitativos. El propósito consistió más bien en compilar, a través de instrumentos estandarizados, datos acerca de la lectura y escritura de los estudiantes para tener una visión global de la asignatura, visión que podría ser contrastada con la recolección de datos y las observaciones realizadas en cada uno de los grupos experimentales.

De esta forma, la metodología estuvo compuesta por estos dos ejes: las secuencias didácticas — a partir del modelo del plan, la acción, la observación y la reflexión- y la estrategia inspirada del cuasiexperimento, con tres grupos experimentales, veinte de control y pruebas de entrada y de salida. La implementación de la investigación se llevó a cabo durante las nueve semanas que duran las asignaturas de Competencias Comunicativas, aunque el desarrollo de las secuencias se dio únicamente durante una o dos semanas de clase, dependiendo del caso de cada una.

\section{RESULTADOS}

Con respecto a Competencias Comunicativas I, se programaron las siguientes secuencias. La primera contó con dos cuestionarios de entrada y dos de salida acerca de la comprensión de una película y de las prácticas de lectura a partir de la producción Un mundo maravi- 
lloso (2006), de Luis Estrada. El objetivo era estimular una visión crítica de la película y la conciencia del estudiante acerca de sus propias prácticas. Luego de los cuestionarios de entrada, los estudiantes debían hacer una ficha de lectura acerca del filme, ficha que incluía la investigación acerca de algunos datos y del contexto de la producción. A partir de estas fichas se realizó un debate en clase y, por último, cerraron la secuencia dos cuestionarios de salida estructuralmente equivalentes a los de entrada. La recolección de datos se llevó a cabo a partir de estos cuestionarios, de las fichas de lectura, de la grabación de la sesión de debate acerca de la película y, finalmente, a partir de una entrevista al cierre de la asignatura realizada por una persona ajena al curso ${ }^{3}$.

Las preguntas acerca de las prácticas de los estudiantes se enfocaron, por ejemplo, en la identificación de lo que se hace después de un ejercicio de lectura, en este caso con un documento audiovisual. Una de las preguntas del cuestionario de entrada era la siguiente: "Después de ver la película ¿consultó alguna información adicional para poder entenderla mejor?". Del cuestionario de salida, una de las preguntas contemplaba la valoración de la búsqueda de información para entender mejor aquello que en una primera lectura puede ser superficial o pasar inadvertido: “¿Conocer información adicional de Luis Estrada y del contexto socio-político de México, le ayudó a comprender mejor la intención del autor?”. Otra se centraba en la valoración personal de la película y en los cambios de percepción después de discutir y socializar los temas de esta con el grupo: "Después del debate sostenido en clase ¿modificaría lo que escribió en el comentario personal de la ficha de lectura?” ". La acogida de la secuencia muestra que la comprensión de un texto o de un video no pasa solo por la capacidad de relacionar elementos locales y por la atención momentánea que se le presta, sino que hay prácticas de lectura que influyen significativamente en la construcción del sentido, y que tienen que ver con el reconocimiento de un contexto sociocultural.

La segunda secuencia para Competencias Comunicativas I se centró en la escritura académica argumentativa a través de tres ejercicios prácticos. El objetivo era mostrarles a los estudiantes, de manera progresiva, cómo se pasa de los textos de opinión personal, comunes en la educación media, a formas académicas impersonales donde se evitan los juicios de valor y, por último, a la escritura académica que expresa una postura, pero con rasgos lingüísticos de impersonalidad y con soporte intertextual para sustentar y argumentar una idea determinada. La recolección de datos consistió en los tres ejercicios escritos, un último texto de percepción de la secuencia, la observación en clase y una entrevista con la practicante de investigación, externa al curso.

En esta secuencia, el primer ejercicio consistió en escribir un texto breve acerca de la apreciación personal de un artículo científico de un área determinada, con la extensión como única restricción. El segundo ejercicio fue precedido de una explicación acerca de ciertas marcas que señalan la pertenencia de los textos a una comunidad discursiva académica y profesional. La explicación se centró en los siguientes rasgos: el uso de un léxico técnico que corresponde a una disciplina dada, de verbos conjugados en tercera persona, de la voz pasiva, de las formas impersonales y de las pasivas reflejas. A partir de esta explicación y del sentido que estos usos tienen en la escritura académica, el segundo ejercicio consistió en realizar un texto de la misma extensión que el anterior, pero esta vez resumiendo solo

La persona a cargo de las entrevistas colectivas de los tres grupos experimentales fue una practicante de investigación, estudiante de último semestre de Lenguas Modernas de la Universidad EAN, Diana Riveros.

4 Las preguntas de estos cuestionarios fueron realizadas por la docente e investigadora Carolina Niño Pantoja. 
el contenido del artículo leído, evitando cualquier tipo de juicio de valor o de opinión al respecto, y siguiendo las pautas dadas del discurso académico. Antes del tercer ejercicio, cada estudiante recibió correcciones y comentarios acerca de sus textos anteriores. Luego, se explicó en clase que en la escritura académica era posible incluir valoraciones, pero que, a diferencia de los textos de opinión, debían estar soportadas por citas textuales o por otras fuentes bibliográficas que apoyaran la postura adoptada frente a lo leído. En el texto académico, la voz propia del estudiante puede expresarse, pero siempre con el apoyo de otras voces de esa comunidad académica, sea a través de un análisis detallado del artículo que se trabaja, sea por medio de la voz de investigadores o autoridades en la materia. Después de esta explicación, cada estudiante redactaba un tercer texto, de la misma extensión y acerca del mismo artículo elegido inicialmente, pero esta vez dando su valoración por medio de los códigos de la escritura académica explicados previamente.

Esta secuencia se inspiró, en parte, en lo que indica Otto Kruse acerca de la escritura académica: "Lo que los estudiantes deben aprender, y lo que probablemente constituye una de las habilidades de escritura más importantes, es un tipo de conciencia textual que les permite amoldarse al discurso académico cuando es necesario, pero también permanecer leal a sus propios modos de pensamiento y escritura" (Kruse, 2003, p. 25). Esa conciencia textual que debe permitirle al estudiante manejar el discurso académico y, a la vez, su propio pensamiento fue el eje de esta secuencia. El paso de un ejercicio de escritura a otro estaba mediado por una explicación de algunas pautas de escritura académica y de su diferencia con la comunicación escrita cotidiana, pero, sobre todo, por el contraste visible para el estudiante entre una forma de escritura y otra.

En cuanto a los resultados, si bien varios estudiantes señalaron la importancia de la comparación de los tres ejercicios y la utilidad de la secuencia, también insistieron en que la redacción del primer texto fue confusa. En efecto, entre el primer y el segundo ejercicio, algunos estudiantes no identificaron con facilidad las diferencias en la escritura, en particular entre la opinión sobre un texto y el hecho de resumirlo. Para algunos, el tipo de texto de opinión y el de resumen resultó siendo muy similar. Esto probablemente habla de una concepción de la escritura por parte de los estudiantes como una suerte de práctica uniforme que mezcla los juicios de valor con la comprensión de los textos, elemento que podría explicar la falta de reconocimiento de lo que algunos géneros específicos argumentativos o expositivos implican en la universidad. Dicho de otra forma, en los primeros semestres se piensa que comprender y opinar acerca de un texto académico corresponden a la misma actividad. Sin embargo, en el tercer ejercicio sí se observó, en todos los casos, un cambio en la forma de aproximarse al escrito, así como un uso concreto y relativamente apropiado de la escritura académica.

Los resultados de esta secuencia didáctica muestran que la concepción de la escritura académica y lo que esta implica no es evidente para los estudiantes de estos cursos, y que necesita una explicación y una mayor práctica. Por último, se constató cómo en el tercer ejercicio, por medio del contraste de los textos propios, las correcciones y las explicaciones del docente, los estudiantes lograron aproximarse a un texto académico en el cual presentan su valoración de manera más rigurosa y clara. Sin embargo, no solo la explicación magistral da cuenta de los resultados: se trata de articular la explicación, la práctica reiterada y contrastiva por parte del estudiante, y la revisión y retroalimentación del docente. Probablemente el conjunto de estos tres elementos ayudó en la creación de esa conciencia textual académica y en el reconocimiento de los cambios en la propia escritura. 
A partir de los resultados de esta actividad, es posible afirmar que no basta con explicar las tipologías de textos y con enseñar los distintos géneros: para transformar la concepción uniforme de la escritura, es fundamental que el estudiante realice el ejercicio del cambio de registro y de género, con una clara conciencia de la finalidad propia de cada uno dentro de un discurso disciplinar dado.

En el caso de Competencias Comunicativas II, la secuencia se centró en el apoyo a la lectura crítica, por medio del análisis de los contextos y de la intertextualidad que cada texto presenta, a partir del uso de textos apócrifos. A partir de un artículo periodístico paródico y apócrifo leído en el aula, se realizaron preguntas acerca de los siguientes aspectos: presupuestos, información pragmática, intenciones ideológicas, información contextual (temporalidad y espacialidad) e intencionalidad. A través de una serie de preguntas, se reveló el carácter apócrifo del texto inicial en el aula.

En un segundo momento, se propuso un nuevo texto apócrifo que fue contrastado con dos fuentes fiables buscadas por los estudiantes previamente, contraste que permitió comparar las diferencias en el tratamiento de la información periodística en ambos casos. Para finalizar, los estudiantes debieron elaborar una consulta posterior acerca de otro tema a partir de tres fuentes, con el propósito de comparar el lenguaje y el tratamiento de la información entre las tres. Al terminar la secuencia, fue posible que los estudiantes identificaran las similitudes y diferencias entre los textos paródicos apócrifos y los textos que tratan la información de forma rigurosa.

Otro de los resultados de esta secuencia, en particular de las preguntas del primer texto apócrifo, da cuenta del poco conocimiento del contexto que tenían los estudiantes respecto a lo leído. El artículo apócrifo trataba acerca de una falsa noticia de actualidad referida a un expresidente de Colombia. Sin embargo, al no tener referentes contextuales, casi ningún estudiante logró identificar inicialmente el carácter falso ni paródico del texto leído. Después de estos ejercicios iniciales, varios estudiantes reconocieron la importancia de buscar fuentes de información fiables y la vulnerabilidad que implica no poder leer críticamente ni reconocer fuentes verídicas de información ${ }^{5}$.

Con respecto a la parte cuantitativa de las pruebas de entrada y de salida, los resultados fueron los siguientes. Cada prueba tenía un componente de lectura y de escritura. En el caso de la lectura, las pruebas consistían en diez preguntas que buscaban establecer distintos niveles a partir de algunos fragmentos de textos o de textos completos. Los niveles fueron los siguientes: uno literal, otro de comprensión y otro crítico. En el caso de la escritura, en el primer nivel de la asignatura se solicitó una reseña crítica, después de explicar brevemente en qué consistía este género; en el segundo nivel se pidió un ensayo argumentativo. En ambos casos se trató de los mismos ejercicios que los estudiantes deben presentar al terminar de cursar la materia, pero en la fase de prueba la nota no hacía parte de la evaluación. De esta forma, se garantizó la uniformidad de los ejercicios de entrada y de salida, aunque el factor de la nota en las pruebas de salida pudo influenciar los resultados. En este orden de ideas, se diseñó una prueba de entrada para Competencias Comunicativas I y otra para el nivel II. De manera paralela, se diseñaron dos pruebas de salida diferentes para cada nivel para un total de cuatro pruebas de lectura distintas. Con respecto a los ejercicios de escritura, se diseñaron dos rúbricas con los criterios de evaluación para la reseña crítica y el ensayo argumentativo. Estas rúbricas fueron usadas por todos los docentes tanto en los

Estas apreciaciones son tomadas de la entrevista llevada a cabo durante la recolección de datos del estudio. 
textos de entrada como en los de salida, con el fin de garantizar cierta uniformidad en la evaluación y así comparar los procesos de los estudiantes.

En cuanto a las pruebas de lectura, las preguntas relacionadas con un nivel literal contemplaban alguna frase o palabra específica y su significado local. Por ejemplo, una de las preguntas literales fue la siguiente:

1. En el enunciado "Sin embargo, este aspecto sólo da cuenta de una parte del complejo fenómeno al que asistimos con la aparición de estas nuevas tecnologías de medios", las palabras subrayadas implican:
a. una comparación entre dos elementos que se han mencionado.
b. una contraposición entre lo dicho anteriormente y lo que se va a decir.
c. una negación de la idea del enunciado inmediatamente anterior.
d. una adición de información relacionada con el enunciado anterior.

A diferencia de estas preguntas, que identificaban un contenido localmente, las preguntas de comprensión partían de una lectura global de los textos y las preguntas de nivel crítico indagaban por la intención comunicativa del autor. Por ejemplo, a partir del texto "El eclipse", de Augusto Monterroso, la siguiente fue considerada una pregunta de comprensión:

9. En el texto se indica que el personaje fray Bartolomé Arrazola "esperó confiado, no sin cierto desdén" porque
a. confiaba en el rey Carlos Quinto y en España.
b. estaba seguro de que los mayas se iban a asustar con el eclipse.
c. creía estar perdido en la selva de Guatemala.
d. tenía confianza en el contenido de los códices mayas.

Sin una comprensión global del sentido del texto, el estudiante no tiene las herramientas para elegir una respuesta acertada. A partir del mismo texto de Monterroso, la siguiente es un ejemplo de pregunta que busca identificar la lectura crítica:

10. En este texto, el autor pretende:
a. describir con precisión la escena de un sacrificio maya.
b. exponer las razones antropológicas de los sacrificios mayas.
c. narrar un encuentro paradójico entre las culturas española y maya.
d. defender la irrelevancia de Aristóteles para los mayas.

El esquema de las cuatro pruebas conservó estos criterios para la elaboración de las preguntas, así como la misma cantidad de ítems. De esta manera, los criterios respaldaron la uniformidad de los instrumentos de evaluación. Además, las cuatro pruebas fueron revisadas por todo el equipo de investigadores hasta ajustarlas y hacerlas lo más uniformes posible en términos de su estructura y de sus contenidos.

Es importante destacar que, dado que la Universidad EAN no tenía una línea de investigación definida vinculada a la escritura académica, no se había usado anteriormente ningún instrumento de evaluación uniforme para todos los grupos de Competencias Comunicativas. Esto implica que el objetivo no era comparar rigurosamente los resultados 
estadísticos con otros esquemas anteriores. Ahora bien, cabe explicar que los programas o syllabus de las dos asignaturas fueron reformulados en su integridad recientemente por el equipo de investigadores, de manera paralela a la realización del estudio. Lo anterior responde al hecho de que, antes de la creación de la línea de investigación, los programas estaban organizados en función de otros temas separados de las habilidades comunicativas como tales. El cambio se hizo de manera simultánea al diseño de este estudio con el propósito de redefinir el objetivo de las asignaturas y poder observar si el cambio tenía resultados positivos en las pruebas de salida; es decir, si el paso por el curso, en su nueva versión, cambiaba el desempeño del estudiante en las pruebas de lectura y escritura de salida propuestas con respecto a las de entrada.

Luego del uso de estas pruebas durante el tercer ciclo académico, los resultados fueron los siguientes. Debido a dificultades administrativas, retiros de asignaturas y contingencias de algunos docentes, hubo una pérdida de datos considerable (contemplando los dos niveles, se perdió un $11,19 \%$ de los datos en la prueba de entrada de lectura; $21,14 \%$ en la de salida; y $19 \%$ en las pruebas de escritura de entrada y salida). Teniendo en cuenta solo los datos válidos, los resultados son los siguientes. Al observar los grupos del nivel I, hubo un aumento en la media de 12,45 puntos en la prueba de lectura (con una desviación típica de 16,42 en la entrada y de 16,83 en la salida) y de 20,53 puntos en la escritura de la reseña crítica (con una desviación típica de 19,27 en la entrada y de 18,14 en la salida). En el nivel II, el aumento en la media fue de 7,29 puntos en la prueba de lectura (con una desviación típica de 15,83 en la entrada y de 16,48 en la salida), y de 21,32 en la escritura (con una desviación típica de 22,08 en la entrada y de 15,04 en la salida).

Para corroborar la existencia de diferencia significativa se realizó la prueba $\mathrm{T}$ para un grupo (tanto en el nivel I como en el II). Se encontró que el sigma es menor a 0.001, por lo que se puede afirmar que hay una diferencia estadísticamente significativa entre la entrada y la salida de los estudiantes que cursaron Competencias Comunicativas I y II. Este es el caso de las pruebas de lectura.

Al reunir los datos de Competencias Comunicativas I y II, la misma prueba $\mathrm{T}$ fue realizada para un grupo. Dado que el sigma también en menor a 0,001, es posible afirmar que tanto en lectura como en la escritura sí hay una diferencia estadísticamente significativa entre la entrada y la salida de los estudiantes de Competencias Comunicativas. La media en lectura en los dos cursos, en una escala de 1 a 100, aumentó globalmente de 62,38 a 71, 38 para las pruebas de lectura, y de 49,95 a 71 para las pruebas de escritura. Este aumento da cuenta de una mejoría en estas habilidades después de cursar estas dos asignaturas.

Las siguientes gráficas ${ }^{6}$ presentan el porcentaje de acierto por tipo de pregunta para las pruebas de lectura de entrada y de salida para el nivel I, con una muestra de 207 datos válidos para la entrada, y de 164 para la salida.

Las gráficas, así como el análisis estadístico de datos fue realizado por la Darcy Milena Barrios Martínez (Mg.), de la Pontificia Universidad Javeriana de Bogotá. 
Gráfico 1. Comparación de prueba de lectura de entrada y salida por pregunta en Competencias Comunicativas I

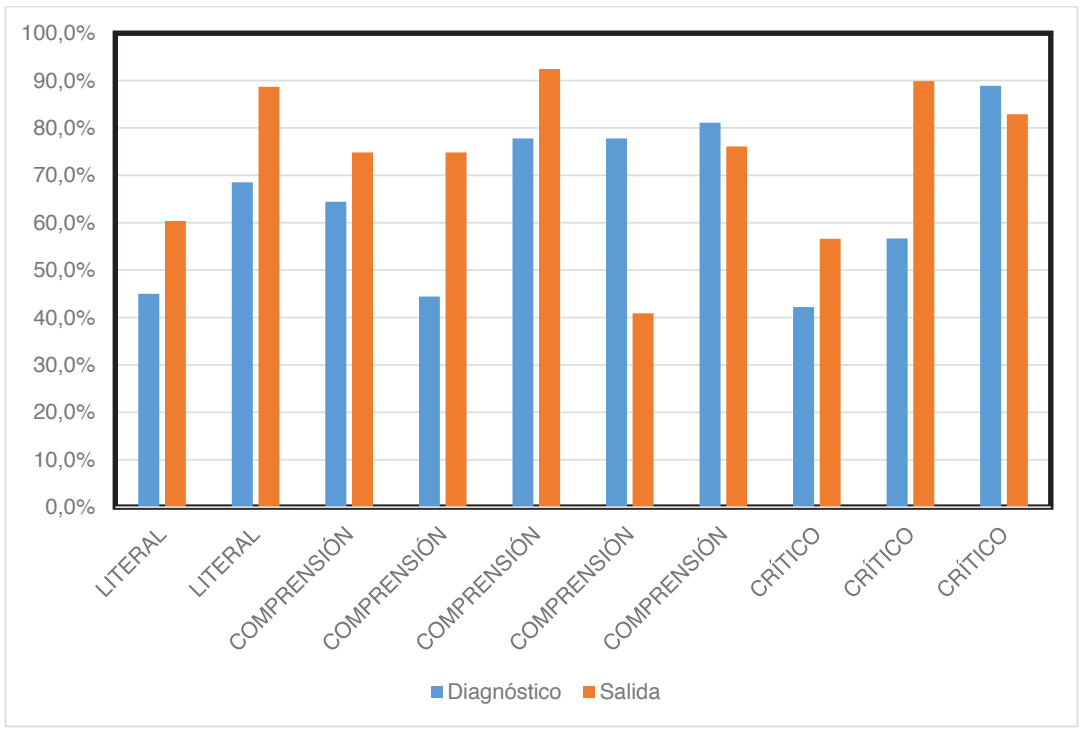

Gráfico 2. Porcentaje promedio de aciertos por tipo de pregunta en Competencias Comunicativas I

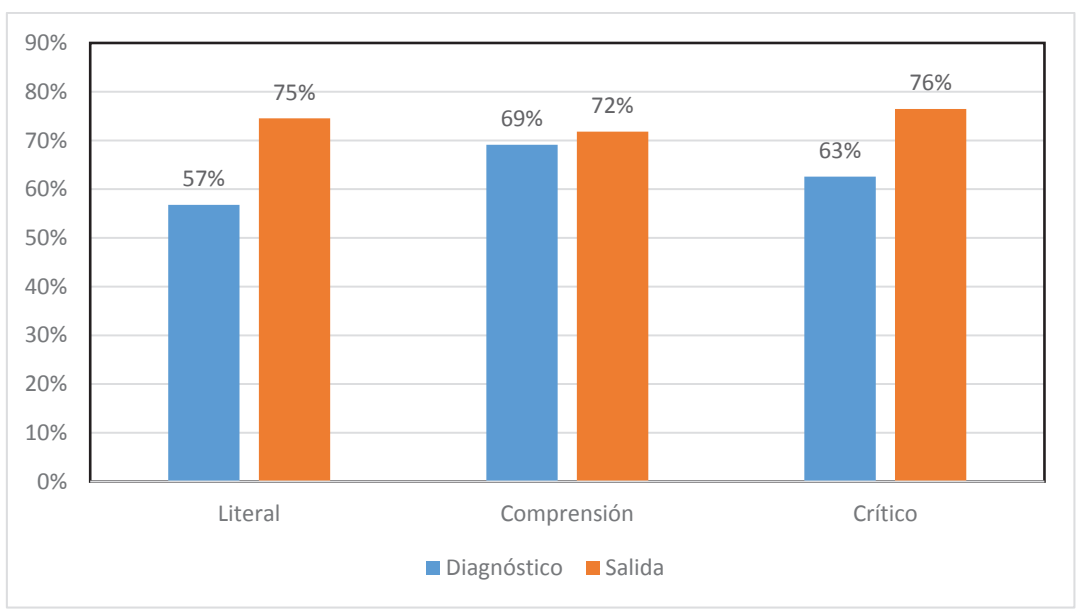

En cuanto al nivel II de la asignatura, las siguientes gráficas muestran la comparación entre el porcentaje de acierto por tipo de pregunta en las pruebas de lectura de entrada y salida, con una muestra de 293 datos válidos para la entrada y 280 para la salida. 
Gráfico 3. Comparación de prueba de lectura de entrada y salida por tipo de pregunta en Competencias Comunicativas II

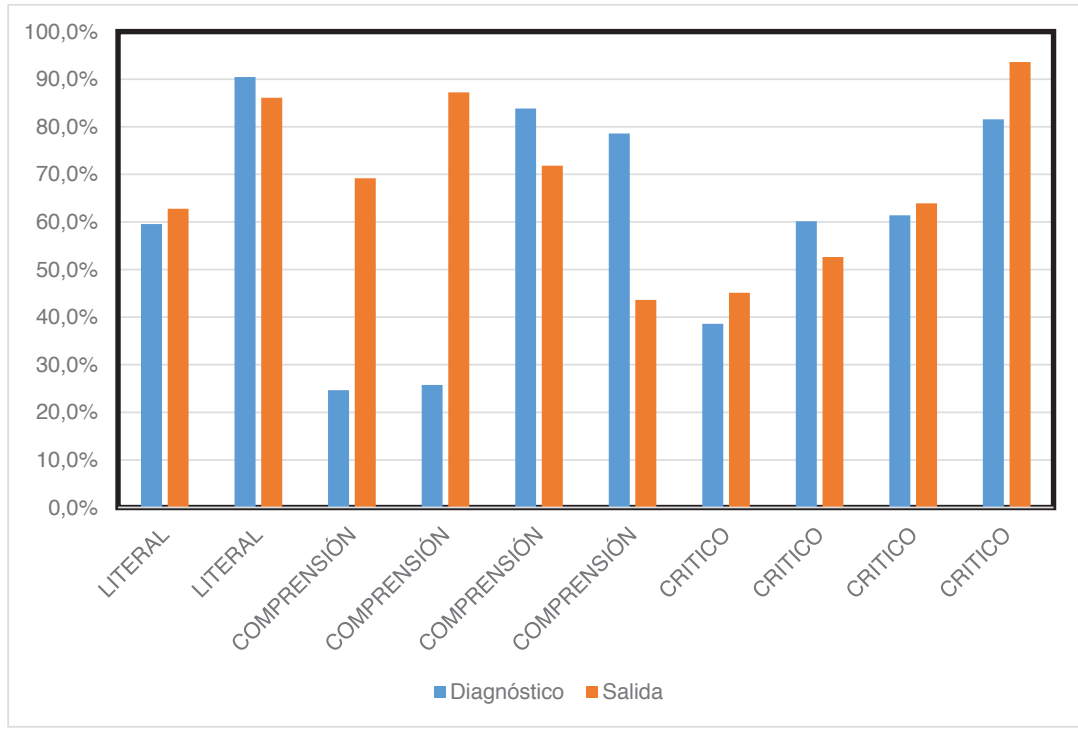

Gráfico 4. Porcentaje promedio de aciertos por tipo de pregunta en Competencias Comunicativas II

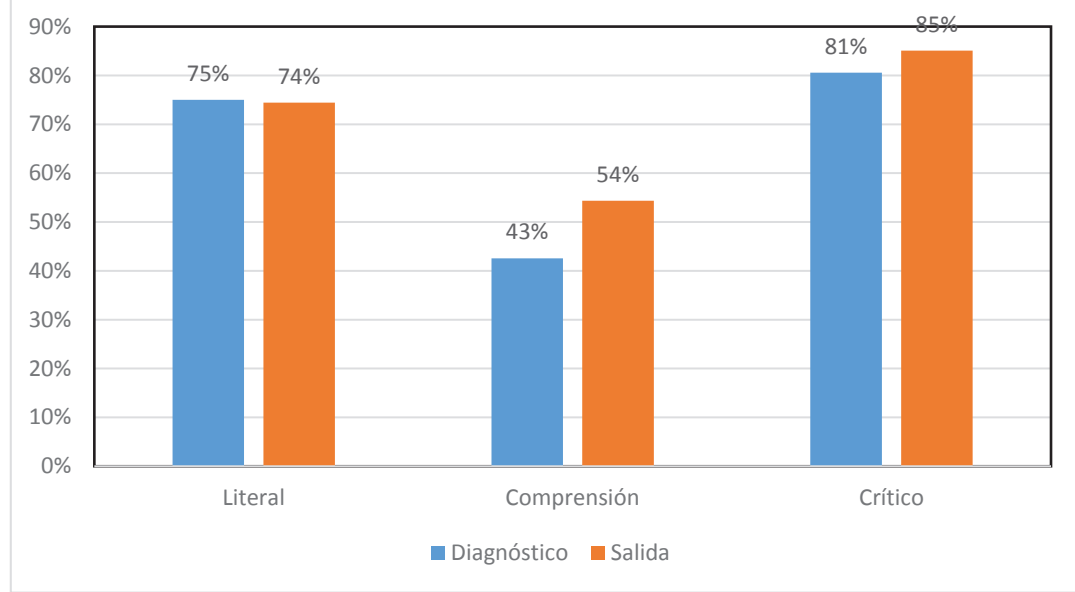

Como se observa en las gráficas, la mejoría en el tipo de preguntas de nivel literal (18\%) y crítico (13\%) es más marcada en el curso de Competencias Comunicativas I, mientras que estos dos niveles cambian menos en Competencias Comunicativas II. De hecho, en las preguntas literales hay un retroceso de $1 \%$. Por otro lado, los estudiantes del segundo 
nivel mejoran en las preguntas de comprensión de manera más notoria (11\%) que los del primer nivel (3\%), aunque el desempeño es inferior al de la asignatura precedente con una diferencia de 18 puntos porcentuales. Es importante señalar en este punto que las pruebas de entrada y salida de Competencias Comunicativas II son de una complejidad mayor que las del nivel I, dado que, si bien las habilidades de lectura y escritura hacen parte del contenido de ambas asignaturas, el segundo nivel tiene un componente de argumentación que implica la comprensión global de textos más complejos. Esta diferencia podría explicar los resultados de los porcentajes por tipo de pregunta. Sin embargo, no deja de ser llamativo el bajo porcentaje en las preguntas de comprensión con respecto a las de lectura literal o crítica. Este resultado parece indicar que, más que dificultades en el reconocimiento de las intenciones de un autor, predominan los obstáculos frente a la comprensión global de los textos, al menos en Competencias Comunicativas II.

\section{CONCLUSIONES}

Con respecto a los resultados cuantitativos, es posible afirmar que el contenido actual de los dos cursos de Competencias Comunicativas es pertinente para la mejora de las habilidades de lectura, escucha, oralidad y escritura. Esto implica que es posible usar esas habilidades como tema y eje central de los cursos con el fin de generar una actitud reflexiva por parte del estudiante frente al lenguaje y, en especial, con respecto al rol de la lectura y la escritura en su propio proceso de aprendizaje. No se trata de concebir la lectura y la escritura como contenidos que estén separados de su forma, ni como formas que se puedan tratar indirectamente a partir de cualquier tema abstracto, como se piensa desde la visión instrumental de estos. Es decir, en esta experiencia fue importante que los cursos trataran explícitamente los temas relacionados con la lectura, la oralidad, la escritura, la escucha, la argumentación y la retórica para que los estudiantes pudieran cambiar la percepción de estas habilidades como algo que ya manejan o que aprendieron en el colegio, y que sería una suerte de instrumento transparente utilizable con cualquier contenido. Si bien este estudio parte de una concepción de la transversalidad de estas habilidades, esto no implica que se defienda una visión instrumental de ellas. Los resultados de las pruebas muestran que hay una mejora en la mayoría de los estudiantes tanto en lectura como en escritura, lo cual permite defender que es importante inculcar una visión no instrumental de estas competencias, que fue la que determinó la reestructuración de los programas llevada a cabo de manera paralela a esta investigación. Las pruebas de entrada y de salida pretendían, además de hacer parte del cuasiexperimento, compilar datos para observar la pertinencia de los nuevos programas.

En este sentido, es fundamental, en primer lugar, propiciar en el estudiante la desnaturalización de estas habilidades y la posterior comprensión del papel central que tienen en la universidad y dentro de cada disciplina. Esta desnaturalización pasa por el reconocimiento de que, en particular leer y escribir, no son una cuestión accesoria e independiente de lo que se ve en la universidad y que se pueda añadir a posteriori frente a un tema cualquiera: menos cuando se trata de cursos de Competencias Comunicativas. El cambio de enfoque en los programas muestra entonces una mejoría observable en los resultados de las pruebas.

De hecho, y es una de las vías para una futura investigación, la visión instrumental de estas habilidades que, en ocasiones, predomina en la educación media y superior, así 
como en ciertos sectores profesionales, puede explicar parcialmente las dificultades de los estudiantes universitarios en el desarrollo de un pensamiento crítico y un carácter reflexivo auténtico frente a sus respectivas disciplinas. Al inculcar solo el pragmatismo y la dimensión práctica y concreta de estas habilidades, es probable que se pierda la noción de su estrecho vínculo con el pensamiento, la racionalidad e, incluso, la creatividad frente a la solución de problemas. En otras palabras, enseñar lectura y escritura solo desde y para fines concretos y prácticos a partir de temas aleatorios podría limitar la conciencia del estudiante frente al papel del lenguaje en el aprendizaje, y generar una consecuente debilidad de su carácter reflexivo y crítico frente a cualquier situación (sea profesional o no). En esta medida, la reformulación de los programas de estas asignaturas, a partir de la reflexión sobre las habilidades comunicativas que están en juego, fue positiva para los resultados generales y el desempeño de los estudiantes en las pruebas realizadas.

Por otra parte, es destacable que estos cursos, al explicitar su rol de facilitadores de herramientas metodológicas para el estudio en la universidad, contribuyen no solo a la mejoría específica en las pruebas de lectura y escritura diseñadas, sino que son un primer acercamiento a otras formas de leer y de escribir que son desconocidas, subestimadas o malinterpretadas hasta el momento por el estudiante neófito. Los resultados muestran que los cursos dan un primer acercamiento a formas de leer y escribir que los estudiantes, en su mayoría, desconocen o que no manejan en la práctica concreta durante su ingreso a la vida universitaria.

En el caso de los grupos experimentales, globalmente no hay una mejoría importante con respecto a los grupos de control. Solo se puede afirmar una diferencia estadísticamente significativa en el caso de la prueba de salida de escritura de las dos asignaturas conjuntas, dado que hay un sigma menor a 0,001 en la prueba T. Sin embargo, y de manera contraria a las expectativas, en este caso la media de los grupos de control es superior (72/100) a la media de los grupos experimentales (65/100). No obstante, puede ser arriesgado atribuir el resultado de estos puntajes exclusivamente a las tres secuencias didácticas llevadas a cabo, dado que hay una multiplicidad de factores que pudieron entrar en juego y que no fueron controlados. Entre ellos, cabe mencionar las diferencias en la práctica individual de cada docente, en la composición de los grupos y en la evaluación de la escritura, a pesar del diseño de las rúbricas.

Ahora bien, a pesar de los resultados estadísticos, las tres secuencias sí dan cuenta de algunas ideas que en ocasiones se dan por sentadas en la docencia: las prácticas de lectura y su relación con la comprensión de textos; la práctica de una escritura académica y argumentativa distinta de la cotidiana; y la necesidad de una serie de referentes contextuales e intertextuales para poder leer críticamente. Estas ideas, que podrían darse por básicas desde la perspectiva docente, parecen ser desconocidas por los estudiantes o, al menos, no tomadas en cuenta cuando ingresan a la universidad, como consta en las entrevistas y en los datos recogidos con cada grupo experimental. Las secuencias didácticas desarrolladas en este estudio enfatizan el señalamiento de estas prácticas como una herramienta metodológica fundamental para leer críticamente y argumentar por escrito, tanto en la universidad como el campo más amplio de la vida personal y profesional. Sin embargo, los estudiantes parecen no estar familiarizados con ellas.

De esta forma, y es la segunda conclusión que podemos plantear a partir de la investigación, aunque no haya sido posible triangular los datos cuantitativos con los datos recogidos durante las secuencias, sí es viable afirmar que estas implican estrategias de 
mejora dado que hacen énfasis en actividades, hábitos o herramientas que los estudiantes desconocen o que no practican. Las experiencias en el aula demuestran que es capital el ejercicio de actividades que progresivamente lleven al estudiante a tomar conciencia de los hábitos, las prácticas, las herramientas y los rasgos de aquello que implica leer críticamente y escribir textos argumentativos en la universidad, a diferencia de sus registros orales y escritos cotidianos, y del aprendizaje en la educación media. Si bien no hay demostración estadística de resultados inmediatos, sí es posible afirmar una mayor conciencia de estas cuestiones a partir de los datos recogidos durante las secuencias.

Para finalizar, cabe indagar si los docentes e investigadores podríamos revisar la concepción del conocimiento que está circulando en las universidades. Probablemente el énfasis en lo práctico y en una educación para la profesionalización como única forma posible del aprendizaje esté colaborando en dejar de lado el desarrollo de un pensamiento crítico que, si bien necesita un contexto y situaciones concretas para emerger, requiere autonomía, adaptación, capacidad de abstracción y creatividad, elementos que no puede ofrecer la simple formación a través de procedimientos para resolver problemas singulares. El aprendizaje de la lectura y de la escritura se da a través de la práctica, porque se trata precisamente de eso, de prácticas; sin embargo, no se trata de prácticas que se aprendan de una vez y para siempre, sino que exigen múltiples habilidades que en cada ocasión intervienen y que juegan un papel fundamental. No son, en ningún caso, procedimientos que tengan lugar sin conceptualización alguna, como sí puede ser el caso de la formación o capacitación de un empleado: “Toda formación se dirige a la adquisición de procedimientos, no a una autonomía general" (Rastier, 2013, p. 26). Por esta razón, el entrenamiento de estas habilidades debe ser amplio y exigente, no limitarse a los formatos o géneros que, se cree, rigen la vida profesional. Para poder desarrollar un pensamiento crítico a partir de la lectura y la escritura, es importante que su práctica incluya una perspectiva más amplia que la de la productividad de cada profesión y que permita, sobre todo, que el estudiante pueda darse cuenta de la complejidad que implica acercarse tanto a la cultura escrita de su disciplina, como al contexto sociocultural en el que está inmerso. Estas prácticas deben recurrir entonces a la estimulación de múltiples capacidades que no tienen que ver solo con la resolución de problemas concretos, sino con el acercamiento a la autonomía, a la complejidad de los textos y, también, del pensamiento y la creatividad.

\section{REFERENCIAS BIBLIOGRÁFICAS}

Adam, J.-M. (2011a). La linguistique textuelle. Introduction à l'analyse textuelle du discours (3a ed.). París: Armand Colin.

Adam, J.-M. (2011b). Les textes, types et prototypes: séquences descriptives, narratives, argumentatives, explicatives, dialogales et genres de l'injonction-instruction (3a ed.). París: Armand Colin.

Benavides, D., \& Sierra, G. (2013). Estrategias didácticas para fomentar la lectura crítica desde la perspectiva de la transversalidad. REICE. Revista Iberoamericana sobre Calidad, Eficacia y Cambio en Educación, 11(3), 79-109.

Björk, L., Bräuer, G., Rienecker, L., \& Jörgensen, P. S. (2003). Teaching Academic Writing in European Higher Education. Dordrecht: Kluwer Academic Publishers.

Campbell, D., \& Stanley, J. (2005). Diseños experimentales y cuasiexperimentales en la investigación social. Buenos Aires: Amorrortu. 
Carlino, P. (2005). Escribir, leer y aprender en la universidad. Una introducción a la alfabetización académica. Buenos Aires: Fondo de Cultura Económica.

Carlino, P. (2011). Leer y escribir en las ciencias sociales en universidades argentinas. Contextos de educación, 14(16), 1-12.

Carlino, P. (2013). Alfabetización académica diez años después. Revista Mexicana de Investigación educativa, 18(57), 355-381.

Carlino, P., \& Fernández, G. (2010). ¿En qué se diferencian las prácticas de lectura y escritura de la universidad y las de la escuela secundaria? Lectura y vida, 31(3), 6-19.

Cassany, D. (2006). Tras las líneas, Sobre la lectura contemporánea. Barcelona: Editorial Anagrama.

Combe, D. (1992). Les genres littéraires. París, Hachette.

Creswell, J. W. (2013). Research Design. Qualitative, Quantitative and mixed Methods Approaches (4th ed.). Thousand Oacks, California: SAGE Publications.

IWCA. (2015). The International Writing Centers Association. Recuperado de http://writingcenters.org

Jones, C., Turne, J., \& Street, B. (1999). Students writing in the University. Cultural and epistemological issues. Amsterdam/Philadelphia: John Benjamins Publishing Company.

Kruse, O. (2003). Getting Started: Academic Writing in the First Year of a University Education. In Björk, L., Bräuer, G., Rienecker, L., \& Jörgensen, P. S. Teaching Academic Writing in European Higher Education (pp. 19-28). Dordrecht: Kluwer Academic Publishers.

Latorre, A. (2008). La investigación-acción. Conocer y cambiar la práctica educativa. Barcelona: Editorial Graó.

McNiff, J. (2013). Action Research. Principles and practice. Oxon: Routledge.

Perelman, C. (1997). El imperio retórico. Bogotá: Editorial Norma.

Perelman, C. (1977). L'empire rhétorique, rhétorique et argumentation. París: Librairie Philosophique J. Vrin.

Perelman, C., \& Olbrechts-Tyteca, L. (1989). Tratado de la argumentación, la nueva retórica. Madrid: Gredos.

Rastier, F. (2013). Apprendre pour transmettre. L'éducation contre l'idéologie managériale. París: Presses Universitaires de France.

RLCPE. (2014). Red Latinoamericana de Centros y Programas de Escritura. Recuperado de https:// sites.google.com/site/redlacpe/

Russell, D. (1990). Writing Across the Curriculum in Historical Perspective: toward a Social Interpretation. College English, 52, 52-73.

Russell, D. (2013). Contradictions regarding teaching and writing (or writing to learn) in the disciplines: What we have learned in the USA. Revista de Docencia Universitaria, 11(1), 161-181.

Vigotsky, L. (1973). Pensamiento y lenguaje. Buenos Aires: Pleyade. 
